\title{
Improved All-Fiber Acousto-Optic Tunable Bandpass Filter
}

\author{
G. Ramírez-Meléndez, M. Bello-Jiménez, O. Pottiez, and M. V. Andrés, Member, IEEE
}

\begin{abstract}
An all-fiber acousto-optic tunable bandpass filter based on a 1.185-mm long coreless core mode blocker is reported. Experimental results demonstrate a minimal insertion loss of 1.2 $\mathrm{dB}$ at the optical resonant wavelength of $1527.7 \mathrm{~nm}$ with 3-dB optical bandwidth of $0.83 \mathrm{~nm}$. The optimization of the device takes into account the attenuation of the acoustic wave and leads to an asymmetric configuration in which the coupling section is shorter than the recoupling part. Under the effect of a standing flexural wave the device can be operated as a bandpass modulator. The device exhibits a maximum modulation depth of $28 \%, 4 \mathrm{~dB}$ of insertion loss and $0.97 \mathrm{~nm}$ of optical bandwidth at 4.774 MHz.
\end{abstract}

Index Terms - Acousto-optic filters, acousto-optic modulation, bandpass filters.

\section{INTRODUCTION}

A COUSTO-OPTIC (AO) interaction based on flexural acoustic waves has been the subject of several research efforts for the development of practical all-fiber AO devices such as frequency shifters, attenuators and tunable filters [13]. Recently, new possibilities have been proposed and demonstrated arising from the use of standing waves $[4,5]$ and short packets of acoustic waves [6]. All these devices offer the advantages of being dynamic devices with tunable amplitude and spectral responses.

The AO effect in optical fibers provides a useful mechanism to bypass a short section of the fiber core $[5,7]$. The acoustic field of flexural waves produces a periodical perturbation of the fiber that leads to an intermodal resonant coupling between the fundamental core mode $L P_{01}$ and some specific cladding modes of the family $L P_{l n}[1,3]$. Thus, the $L P_{01}$ mode can be coupled to a cladding mode to avoid an obstacle located at the fiber core via cladding propagation, later this cladding mode can be coupled back to the core mode at the end of the

This investigation has been financially supported by CONACyT grant 222476.

G. Ramírez-Meléndez and M. Bello-Jiménez are with the Instituto de Investigación en Comunicación Óptica, Universidad Autónoma de San Luis Potosí, Av. Karakorum 1470, Lomas 4ta Secc., San Luis Potosí, S.L.P, México (e-mail: gustavo_r_melendez@hotmail.com; m.bello@ cactus.iico.uaslp.mx).

O. Pottiez is with the Centro de Investigaciones en Óptica (CIO), Loma del Bosque 115, Colonia Lomas del Campestre, 37150 León, GTO, México (email: pottiez@cio.mx).

M. V. Andrés is with the Departamento de Física Aplicada y Electromagnetismo, Universidad de Valencia, c/Dr. Moliner 50, E- 46100 Burjassot, Valencia, Spain (e-mail: miguel.andres@uv.es). interaction length. Under this configuration the device operates as a bandpass filter, and the light whose wavelength does not fulfill the phase-matching condition is strongly attenuated. All-fiber AO tunable bandpass filters (AOTBFs) based on this mechanism have been proposed and demonstrated in which the obstacle is composed of a core mode blocker (CMB) [5, 8-11]. CMBs have been fabricated by core damage $[8,9]$, specially designed hollow optical fibers [10], and ultraviolet (UV)-induced effect [11]. From these devices, a maximum suppression for the non-resonant modes of $-35 \mathrm{~dB}$ can be achieved with a CMB made of local damage in the core [8], and a minimum insertion loss of $1 \mathrm{~dB}$ in a bandpass configuration based on UV-induced CMB [11].

In this letter we propose an AOTBPF that combines the dynamic properties of the $\mathrm{AO}$ interaction with a $\mathrm{CMB}$ composed of a tiny section of coreless optical fiber. Compared with previous AOTBFs our approach has the advantage of avoiding complicated procedures for CMB fabrication. In addition, the inclusion of a coreless optical fiber avoids reflections of the acoustic wave and does not perturb the cladding modes. With the proposed scheme a maximum rejection efficiency of $-31 \mathrm{~dB}$ is obtained at the optical wavelength of $1545 \mathrm{~nm}$. In addition, taking into account the attenuation of the acoustic wave, which is usually neglected, we demonstrate that it is possible to optimize the response by adjusting properly the coupling and recoupling interaction lengths. With the objective of expanding the application of the AOTBF to an acousto-optic bandpass modulator (AOBM), acoustic reflection was intentionally induced. Thus, a standing acoustic wave is generated which produces amplitude modulation at twice the acoustic frequency.

\section{THE AO TUNABLE BANDPASS FILTER}

The scheme of the AOTBF is depicted in Fig 1. It consists of a CMB composed of a tiny section of coreless optical fiber (Thorlabs FG125LA) spliced between two sections of standard single-mode fibers (SMF-28) with lengths $L_{1}$ and $L_{2}$, respectively. The length $d$ of the CMB is calculated for the light leaving section $L_{l}$ to be expanded up to the outer surface at the front face of the following single-mode fiber. Assuming an effective index of 1.446 for the $L P_{01}$ mode and a refractive index of 1.444 for the coreless fiber, the distance $d$ is calculated to be $1.185 \mathrm{~mm}$. With this configuration, the fundamental mode is strongly attenuated in a broadband that extends from 1520 to $2570 \mathrm{~nm}$ with a maximum attenuation of $-31 \mathrm{~dB}$ at $1545 \mathrm{~nm}$. The AOTBF is completed with the radio- 
frequency (RF) source, a piezoelectric disk, and an aluminum horn. The horn is attached to the piezoelectric disk, and it focuses the vibrations into the optical fiber through its dip, which is glued to an uncoated optical fiber to prevent the attenuation of the acoustic wave. In order to avoid acoustic reflections, the acoustic wave is damped at both ends of the filter structure.

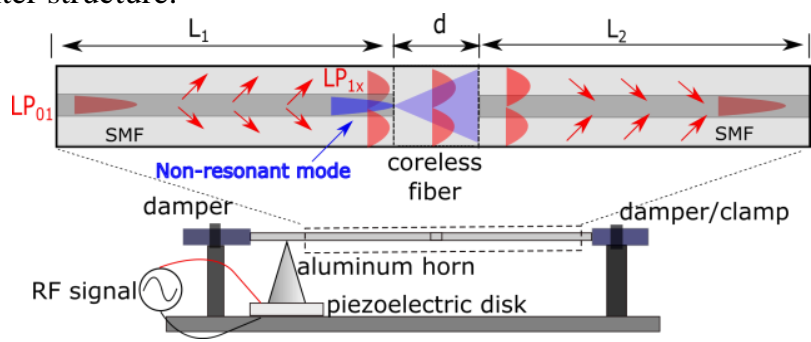

Fig. 1. Experimental setup of the AOTBF based on a coreless optical fiber CMB.

When a flexural acoustic wave propagates along the uncoated optical fiber, the acoustic field produces a periodical perturbation of the fiber, which leads to an intermodal resonant coupling between the fundamental core mode and some specific cladding modes of the SMF [1,3]. This AO interaction can be seen as the dynamic counterpart of a conventional long-period grating (LPG), whose transmission properties can be controlled dynamically by the amplitude and frequency of the acoustic wave. In our approach, the section of fiber $L_{l}$ was set to $24 \mathrm{~cm}$ in length, allowing the $L P_{0 l}$ mode to be coupled to a cladding mode prior the $\mathrm{CMB}$, whereas section $L_{2}$ is used to couple the light back to the core mode. This reinsertion of energy results in the appearance of a transmission notch in the spectrum, with an amplitude that remains fixed whenever the travelling acoustic wave is excited. Under this scheme the device operates as a bandpass filter, and wavelengths that do not fulfill the phase-matching condition are strongly attenuated. For the first set of experiments, the length of section $L_{2}$ was selected to be the same as $L_{l}(24 \mathrm{~cm})$, giving a total length of $\sim 48 \mathrm{~cm}$ for the AO device.

The transmission properties of the AOTBF were investigated by illuminating the device with a superluminescent diode light source (SLD), that provides an optical spectrum from 1500 to $1600 \mathrm{~nm}$, and detecting the transmitted light with an optical spectrum analyzer. In order to achieve maximum efficiency, a polarization controller was inserted to control the polarization state of the light entering the AOTBF. The strongest mode-coupling was found at the acoustic frequency $\left(f_{a}\right)$ of $2.384 \mathrm{MHz}$ for a voltage applied to the piezoelectric disk $\left(V_{P D}\right)$ of $33 \mathrm{~V}$ (whenever we refer to voltages, it is a peak-to-peak measurement). Beyond this voltage the response of the piezoelectric disk degrades due to an excessive heating. Our experiments were carried out using a maximum voltage of $33 \mathrm{~V}$, where a stable and linear response of the piezoelectric was observed. At this voltage the measured RF power into the device was $1.4 \mathrm{~W}$. Fig. 2(a) shows the transmission spectrum, in which $L P_{01}$ to $L P_{11}$ and $L P_{01}$ to $L P_{12}$ intermodal couplings were observed with a wavelength separation of $\sim 50 \mathrm{~nm}$. The maximum transfer of energy corresponds to the $L P_{01}$ to $L P_{11}$ coupling located at the resonant optical wavelength $\left(\lambda_{R}\right)$ of $1527.7 \mathrm{~nm}$, with a measured 3-dB optical bandwidth of $1.2 \mathrm{~nm}$ and $-2.78 \mathrm{~dB}$ insertion loss. The period of the acoustic LPG is estimated to be $688.77 \mu \mathrm{m}$, obtained from the dispersion relation for a flexural acoustic wave on a cylindrical $\operatorname{rod} \Lambda=\sqrt{ }\left(\pi R C_{e x} / f_{a}\right)$, where $\Lambda$ is the acoustic wavelength, $R$ is the fiber radius and $C_{e x t}$ is the speed of the extensional wave $\left(5760 \mathrm{~ms}^{-1}\right.$ for fused silica).

Experimental characterization of the $\mathrm{AO}$ resonances is shown in Fig. 2(b) for a range of resonant optical wavelengths between 1520 and $1600 \mathrm{~nm}$. Working in the frequency range from 2 to $2.8 \mathrm{MHz}, L P_{01}$ to $L P_{11}, L P_{01}$ to $L P_{12}$, and $L P_{01}$ to $L P_{13}$ intermodal couplings are observed. The strongest mode coupling was found at the acoustic frequency of $2.384 \mathrm{MHz}$ (dashed line).
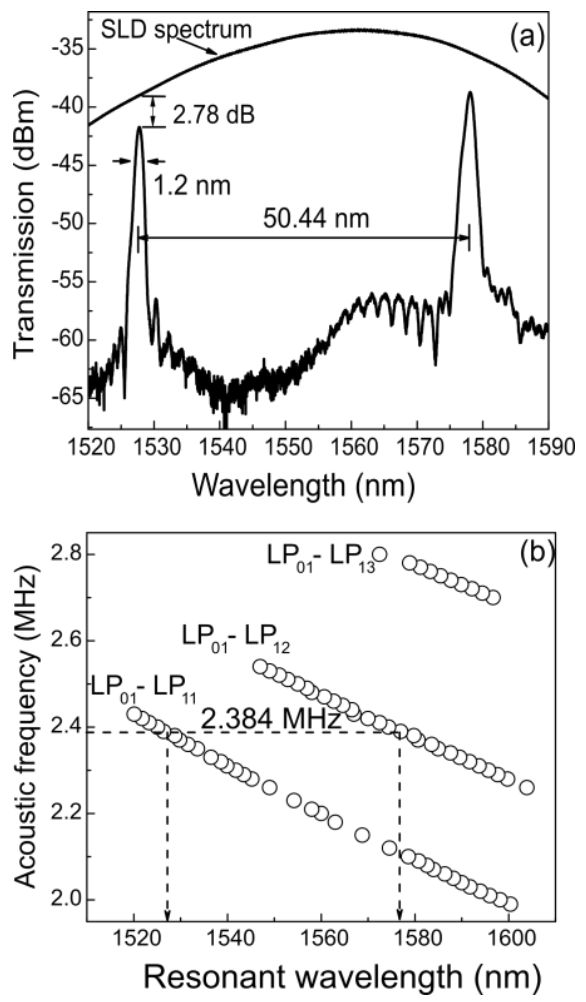

Fig. 2. (a) Transmission spectrum of the AOTBF at the acoustic frequency of $2.384 \mathrm{MHz}$. (b) Tuning characteristics of the AOTBF: acoustic wave frequency vs resonant optical wavelengths. The dashed line indicates the strongest mode coupling.

An important characteristic of our proposed scheme is the relatively long interaction length, which is of several tens of centimeters. Therefore, the effect of acoustic attenuation is an important factor to improve filter performance. The effect of acoustic attenuation leads to a reduction of the coupling coefficient $\kappa$, which is proportional to the acoustic amplitude. At the resonant wavelength $\left(\lambda_{R}\right)$ the transmission of light in the $L P_{01}$ mode as a function of length $L$ can be expressed by

$$
T_{\lambda_{R}}=1-\sin ^{2}\left(\kappa_{0} \cdot e^{-\alpha L} L\right)
$$


where $\kappa_{0}$ is the coupling coefficient at a distance $L=0$ and $\alpha$ is the attenuation coefficient of the acoustic wave in the fiber. From this equation it is clear that a total transfer of energy from the fundamental mode to one of the cladding modes is obtained when the argument $\kappa_{0} e^{-\alpha \mathrm{L}} L$ reaches the value of $\pi / 2$, and conversely when it reaches the value of $\pi$. In order to achieve the optimal interaction length, the total length $(L)$ of the AOTBF was varied from 25 to $90 \mathrm{~cm}$. These measurements were performed maintaining fixed the distance $L_{1}$, equal to $24 \mathrm{~cm}$, ensuring the maximum coupling between the core and cladding modes prior the CMB. Fig. 3 shows the filter transmission as a function of length for the $L P_{01}$ to $L P_{11}$ mode coupling at the resonant wavelength of $1527.7 \mathrm{~nm}$. The acoustic frequency and the voltage applied to the piezoelectric was fixed at $2.384 \mathrm{MHz}$ and $33 \mathrm{~V}$, respectively. From these results a rough estimation of $\alpha$ can be obtained considering maximum $(L=70 \mathrm{~cm})$ and minimum $(L=24 \mathrm{~cm})$ values of the transmission curve. We obtain a measurement of $\alpha=0.75$ $\mathrm{m}^{-1}$. This value is consistent with the reported value of 0.66 $\mathrm{m}^{-1}$ in [6] for the SMF-28 fiber.

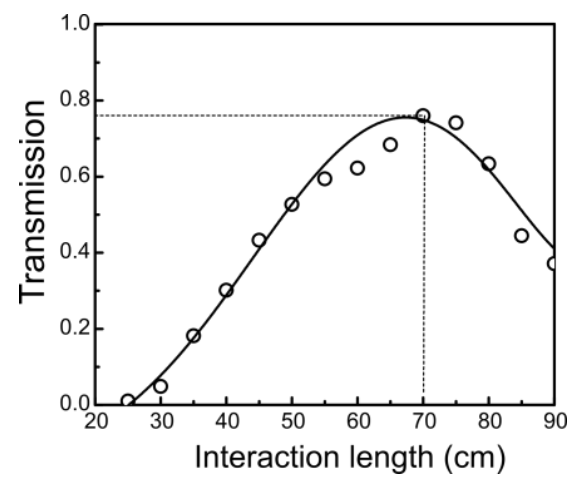

Fig. 3. Filter transmission as function of length for the $L P_{01}$ to $L P_{11}$ mode coupling at the resonant optical wavelength of $1527.7 \mathrm{~nm}$. The acoustic frequency and the voltage applied to the piezoelectric were fixed at 2.384 $\mathrm{MHz}$ and $33 \mathrm{~V}$, respectively.

From these results it can be observed that transmission reaches its maximum value at the length of $L=70 \mathrm{~cm}$, at this length there is a $76 \%$ reinsertion of light for the resonant optical wavelength. Fig 4 shows the transmission spectrum obtained with $L$ equal to $70 \mathrm{~cm}$ and an asymmetric configuration: $L_{1}=24 \mathrm{~cm}$ and $L_{2}=46 \mathrm{~cm}$. A minimum insertion loss of $-1.2 \mathrm{~dB}$ was measured at the resonant wavelength of $1527.7 \mathrm{~nm}$ with a $3-\mathrm{dB}$ optical bandwidth of $0.83 \mathrm{~dB}$. From this result we emphasize the low insertion loss of $\sim 1 \mathrm{~dB}$ and the easy implementation of the CMB. If we compare these results with similar reported schemes of AOTBFs [8-11], we can appreciate the improvement achieved with the present scheme.

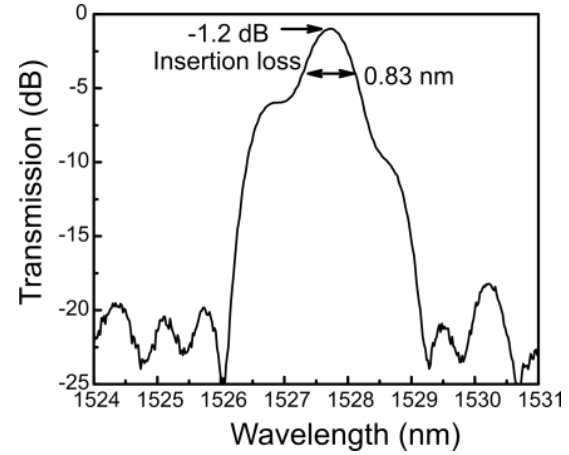

Fig. 4. Transmission spectrum of the AOTBF at the optimal length of $L=70$ $\mathrm{cm}$ and the acoustic frequency of $2.384 \mathrm{MHz}$.

\section{THE AO EFFECT USING A STANDING FLEXURAL WAVE}

With the objective of expanding the application of the AOTBF to an acousto-optic bandpass modulator (AOBM), acoustic reflection was intentionally induced. By firmly clamping one end of the fiber, opposite to the aluminum horn, a standing flexural acoustic wave can be generated, and the transmission of the AOTBF can be converted into a bandpass AO modulation. In this case, the device exhibits a transmission which oscillates in time as a result of the instantaneous perturbation generated by the standing flexural wave. Hence, the transmission is amplitude modulated at a frequency two times the frequency of the acoustic wave. Fig. 5(a) shows the transmitted light as a function of time when a $\mathrm{RF}$ signal of $2.384 \mathrm{MHz}$ and $28 \mathrm{~V}$ is applied to the piezoelectric disk. The maximum modulation amplitude occurs at the resonant optical wavelength of $1526.8 \mathrm{~nm}$. An amplitude modulation at $4.774 \mathrm{MHz}$ is clearly visible, which is two times the acoustic frequency used $(2.384 \mathrm{MHz})$. The measurements reported in Fig. 5 were performed by illuminating the modulator with a tunable laser diode around the resonant optical wavelength, and detecting and monitoring the transmitted light with a standard oscilloscope. The fact that the reflection coefficient for the acoustic wave is not 1 , in conjunction with acoustic losses, causes the maximum transmission to be below the reference level, i.e., the transmission of the fiber when there are no acoustic wave and $\mathrm{CMB}$. Therefore, the maximum transmission determines the insertion loss of the modulator and the difference between the maximum transmission and the minimum determines the modulation depth. From the results presented in Fig. 5(a) we can measure a modulation depth of $28 \%$, together with a low insertion loss of $-4.07 \mathrm{~dB}$.

For applications, the modulator has a number of specific characteristics that require to be properly analyzed. First, we measured the modulation depth as a function of the optical wavelength, when both the acoustic frequency and the RF voltage were fixed. Fig. 5(b) shows the modulation dependence around the resonant wavelength of $1526.8 \mathrm{~nm}$ (2.384 MHz and $28 \mathrm{~V}$ as in Fig. 5(a)). At the resonant optical wavelength the modulation depth is maximal, and symmetrically decreases for longer and shorter wavelengths. The measured $-3 \mathrm{~dB}$ bandwidth is $0.97 \mathrm{~nm}$, with a maximal 
modulation depth of [30\%]. On the other hand, an acoustic resonator is also formed. Fig. 5(c) shows the modulation depth versus the detuning frequency $\left(\Delta f_{a}\right)$ when $\lambda_{R}$ and $V_{P D}$ are fixed at $1526.8 \mathrm{~nm}$ and $28 \mathrm{~V}$, respectively. The center frequency in Fig. 5(c) corresponds to $2.384 \mathrm{MHz}$, at this frequency the modulation depth is maximal, and it drops gradually to values close to zero for frequencies of around $\pm 2.5 \mathrm{kHz}$ and reaches a second maximum at $\pm 3 \mathrm{kHz}$. For longer and shorter frequencies the transmission decays gradually to zero. Therefore, the proper operation of the AOBM is determined by the acoustic frequency $f_{a}$, which needs to be properly selected to achieve the maximum modulation depth.

From the point of view of implementation, the AOBM is well suited for active mode-locking. The AOBM can be dynamically controlled by the acoustic wave, providing a finetuning of the acoustic frequency to match the cavity round-trip time with the modulation period and no frequency shift is produced. Additionally, with the use of this modulator, the operation of the mode locker is simplified since no additional filtering devices are necessary due to the bandpass characteristics of the modulator. Further improvements in efficiency and interaction length could be obtained by including tapered optical fiber in the modulator.
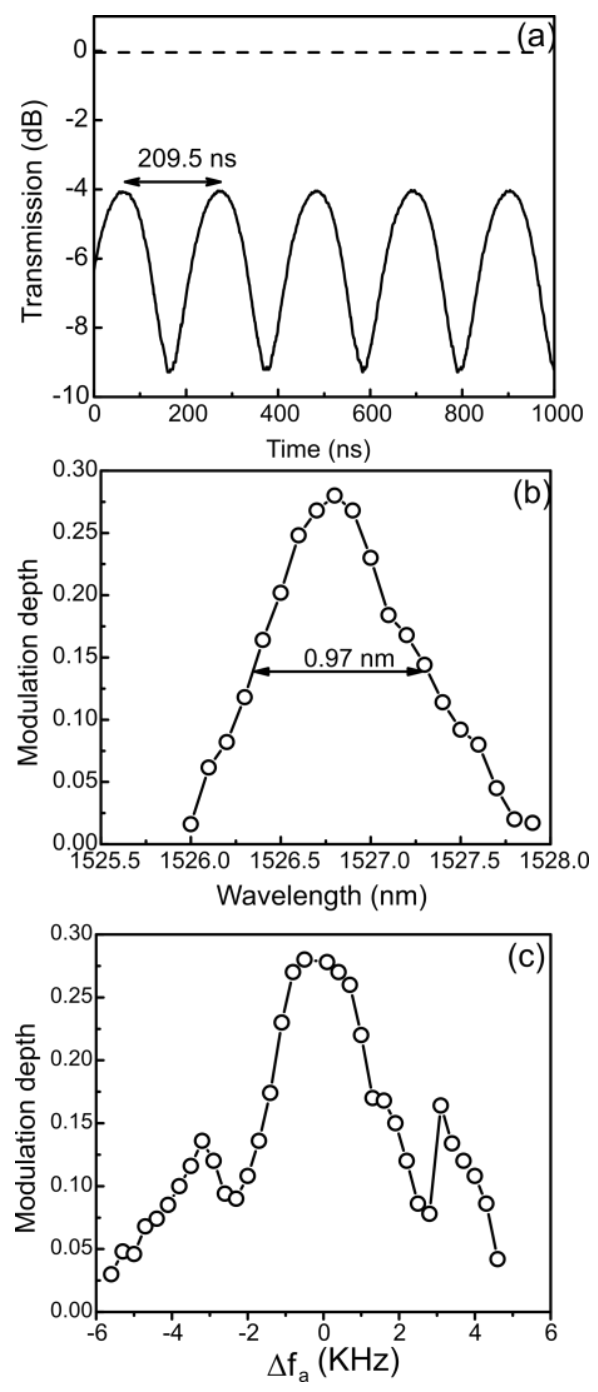

Fig. 5. (a) Oscilloscope trace of the AO amplitude modulation recorded at the resonant wavelength of $1526.8 \mathrm{~nm}$. (b) Modulation depth as a function of the optical wavelength at constant acoustic frequency $(2.384 \mathrm{MHz})$ and $\mathrm{RF}$ voltage $(28 \mathrm{~V})$. (c) Modulation depth as a function of the detuning frequency $\left(\Delta f_{a}\right)$ when both the resonant optical wavelength $(1526.8 \mathrm{~nm})$ and the RF voltage $(28 \mathrm{~V})$ are fixed. The central acoustic wave in Fig. 5(c) is $2.384 \mathrm{MHz}$.

\section{CONCLUSION}

We have demonstrated an all-fiber acousto-optic tunable bandpass filter based on a coreless optical fiber core mode blocker. The response is optimized with an asymmetric configuration that takes into account the attenuation of the acoustic wave. Experimental results demonstrate a lowest insertion loss of $-1.2 \mathrm{~dB}$ at the optical resonant wavelength of $1527.7 \mathrm{~nm}$ with $-3 \mathrm{~dB}$ optical bandwidth of $0.83 \mathrm{~nm}$ when the device is operated as a bandpass filter. Under the effect of a standing flexural wave the AO device can be operated as a bandpass modulator. The device exhibits an amplitude modulation at $4.774 \mathrm{MHz}$, which is two times the acoustic frequency used $(2.384 \mathrm{MHz})$. The results demonstrate a maximum modulation depth of $28 \%,-4 \mathrm{~dB}$ of insertion loss and $0.97 \mathrm{~nm}$ of modulation bandwidth.

\section{REFERENCES}

[1] S. D. Lim, J. G. Kim, K. Lee, S. B. Lee and B. Y. Kim, "All-fiber acousto-optic frequency shifter," Opt. Lett., vol. 11, no. 6, pp. 389-391, June 1986.

[2] D. Östling and ,H. Engan, "Narrow-band acousto-optic tunable filtering in a two-mode fiber," Opt. Lett. vol. 20, no. 11, pp. 1247-1249, June 1995.

[3] T. A. Birks, P. St. J. Russel and D. O. Culverhouse, "The acousto-optic effect in single-mode fiber tapers and couplers," J. Lightwave Technol., vol. 14, no. 11, pp. 2519-2529, Nov. 1996.

[4] M. Bello-Jiménez, C. Cuadrado-Laborde, D. Sáez-Rodríguez, A. Díez, J. L. Cruz, and M. V. Andrés, "Actively mode-locked fiber ring laser by intermodal acousto-optic modulation," Opt. Lett., vol. 35, no. 22, pp. 3781-3783, Nov. 2010.

[5] C. Cuadrado-Laborde, M. Bello-Jiménez, A. Díez, J. L. Cruz, and M. V. Andrés., "Long-cavity all-fiber ring laser actively mode locked with bandpass acousto-optic modulator," Opt. Lett., vol. 39, no. 1, pp. 68-71, Jan. 2014.

[6] E. P. Alcusa-Sáes, A. Díez, M. González-Herráez, and M. V. Andrés, "Time-resolved acousto-optic interaction in single-mode optical fibers: characterization of axial nonuniformities at the nanometer scale," Opt. Lett., vol. 39, no. 6, pp. 1437-1440, March 2014.

[7] A. Díez, M. Delgado-Pinar, J. Mora, J. L. Cruz, and M. V. Andrés, "Dynamic fiber-optic add-drop multiplexer using bragg gratings and acousto-optic-induced coupling," IEEE Photon.Technol. Lett., vol. 15, no. 1, pp. 84-86, Jan. 2003.

[8] T. Dimmick, D. A. Satorius, and G. L. Burdge, "All-fiber acousto-optic tunable bandpass filter," Optical Fiber Communication Conference and International Conference on Quantum Information, 2001 OSA Technical Digest Series (Optical Society of America, 2001), paper WJ3.

[9] D. A. Satorius, T. E. Dimmick, and G. L. Burdge,“ Double-pass acoustooptic tunable bandpass filter with zero frequency shift and reduced polarization sensitivity," IEEE Photonics Technology Letters, vol. 14, issue 9, pp. 1324-1326.

[10] Y. Jung, S. Choi, Y. S. Jeong, S. B. Lee, J. W. Yu, and K. Oh, "Hollow optical fiber core mode blocker for acousto-optic bandpass filter" in CLEO, 2004, paper CThMM6.

[11] K. J. Lee, D. II Yeom, and B. Y. Kim, "Narrowband, polarization insensitive all-fiber acousto-optic tunable bandpass filter," Opt. Express, vol. 15, no. 6, March 2007. 